\title{
Introduction to the Minitrack on Digital-Enabled Human-Information Interaction
}

\author{
Aylin Imeri \\ Heinrich Heine University Düsseldorf \\ aylin.imeri@hhu.de
}

\section{Motivation}

Access to information through our digital world enables various entry points, starting with an initial google search to different social media platforms. Therefore, according to Ottosen, Mani, and Fratta [1, p. 210], "today's information environment has shifted from one of patron dependence on libraries and librarians as gatekeepers to information and knowledge to one self-driven information seeking."

Already Johnson and Case [2, p. 5] call the attention that today's information environment is characterized "through the explosive growth of information coupled with the ready access instantiated in the Internet." Even if this was about nine years back, research on digitalenabled human-information interaction is still growing. The explosive growth requires even more (health) information literate people. Ottosen, Mani, and Fratta [2] reveal that digitalization enables, on the one hand, omnipresent accessible content but, on the other hand, situations where people need to critically reflect on the quality, reliability, and usefulness of that information.

According to McCay-Peet and Quan-Haase [3, p. 13], social media "has become an integral part of everyday life with large economic, political, and societal implications." Social media research enables us to understand a variety of aspects based on social media data. For one example, "[s]ocial media activity is the focus of research in this case (e.g., how people discuss issues related to personal health on Facebook, topological features of Twitter networks, and social media usage patterns)" [3, p. 19].

Individuals also use social media to share experiences, receive, and enable social, informational, and emotional support within Reddit [4]. Social media research within the health-related domain focuses on different topics, such as chronic disease (e.g., diabetes, heart disease) $[5,6,7]$ but also on infectious disease outbreaks, such Swine flu $[8,9]$, and in the last years on COVID-19 [10,11,12, 13, 14].
Social Media research within the health-related domain enables understanding users' information behavior and practices and raising awareness for the produced and shared content itself (sources, quality, truthfulness). Psychological circumstances could affect the information-seeking behavior as well as the use and production of information. Psychological theories such as confirmation bias theory [15] or affective responses (e.g., information anxiety) can also influence users' human-information interaction.

Based on this compressed introduction, this minitrack tried to bring researchers from different disciplines together and enable insights from different perspectives, both methodical and conceptual. This should lead to a vibrant discussion regarding social media usage and users' digital information interaction to understand the impact of health-related topics on everyday life.

\section{Social Media Research and COVID-19}

In this minitrack, the two presentations are thematically concentrating on COVID-19 at least its influence on users' (information) behavior during the COVID-19 pandemic.

The paper Coping and Regulatory Responses on Social Media during Health Crises: A Large-scale Analysis [16] enables insights into a theoretical concept, the applied coping and regulatory behavior.

According to Skinner and Zimmer-Gembeck [17] "coping is an adaptive process, integral to functioning and development. At its most general, coping depicts how people detect, appraise, deal with, and learn from actual and anticipated stressful encounters." Abramova, Batzel, and Modesti [16] investigated the information exchange on Twitter (around $800 \mathrm{~K}$ German tweets) by analyzing the emotion-related and problem-related reactions to better understand the response to health crises. Thereby they are applying a framework consisting of fours aspects, namely (1) Understanding 
(problem-promotion), (2) Action planning (problemprevention), (3) Hope (emotion-promotion), and (4) Reassurance (emotion-prevention). Topic extraction within this investigation was done with unsupervised machine learning approach (structural topic model (STM)).

Knittel and Wash [18] decided to investigate How an Online Health Support Community Responded to the COVID-19 Crisis. While the first paper concentrated on Twitter, here Reddit, the anonymous social media platform, is focused. To what extent does a health crisis such as the COVID-19 pandemic affect the information seeking and crisis communication for health-related information and the structural and social changes within a social community? In this paper, the subreddit $r$ /infertility is selected to understand to what extent structural changes are identifiable. Further, the authors investigate to what extent a health crisis, such as COVID-19 impacts the balance between COVID-19 related needs and the goals regarding living with infertility? The authors applied an inductive qualitative content analysis.

To wrap up, this minitrack enables valuable insights into two different social media platforms (anonymous vs. non-anonymous and microblogging vs. discussion forum) by conducting two different methods (machine learning approach and qualitative content analysis).

\section{Acknowledgements}

I would like to thank all authors for considering submitting their valuable work to this minitrack. I also would like to thank the reviewers for their constructive reviews and support.

\section{References}

[1] T. Ottosen, N.S. Mani, and M.N. Fratta, "Health information literacy awareness and capacity building: Present and future", IFLA Journal, July 10, 2019, pp. 207-215, https://doi.org/10.1177/0340035219857441

[2] Johnson, J.D., and D.O. Case, Health Information Seeking, Peter Lang Publishing, New York, NY, 2012.

[3] McCay-Peet, L., and A. Quan-Haase, "What is social media and what questions can social media research help us answer?", in L. Sloan, and A. Quan-Haase (eds.), The SAGE Handbook of Social Media Research Methods, SAGE, 2017, pp. 13-26.

[4] M.D. Choudhury, and S. De, "Mental health discourse on Reddit: Self- disclosure, social support, and anonymity", in Proceedings of the 8th International Conference on Weblogs and Social Media, June 2014, pp. 71-80

[5] J.A. Greene, N.K. Choudhry, E. Kilabuk, and W.H. Shrank, "Online social networking by patients with diabetes: A qualitative evaluation of communication with Facebook", Journal of General Internal Medicine, October 13, 2020, pp. 287-292, https://doi.org/10.1007/s11606-010-1526-3

[[6] A. Karami, A.A. Dahl, G. Turner-McGrievy, H. Kharrazi, and G. Shaw Jr., "Characterizing diabetes, diet, exercise, and obesity comments on Twitter", International Journal of Information Management, February 2018, pp. 1-6, https://doi.org/10.1016/j.ijinfomgt.2017.08.002

[[7] S.R. Partridge, P. Gallagher, B. Freeman, and R. Gallagher, "Facebook groups for the management of chronic diseases", Journal of Medical Internet Research, January 2018, e21, https://doi.org/10.2196/jmir.7558

[[8] C. Chew, and G. Eysenbach, "Pandemics in the age of Twitter: content analysis of Tweets during the 2009 H1N1 outbreak", PLoS One, Nov 29, 2010, e14118, https://doi.org/10.1371/journal.pone.0014118

[[9] W. Ahmed, P.A. Bath, L. Sbaffi, and G. Demartini, "Novel insights into views towards H1N1 during the 2009 Pandemic: A thematic analysis of twitter data", Health Information and Libraries Journal, January 20, 2019, pp. 60-72, https://doi.org/10.1111/hir.12247

[[10] C. Huang, X. Xu, Y. Cai, Q. Ge, G. Zeng, X. Li, W. Zhang, C. Ji, and L. Yang, "Mining the characteristics of COVID-19 patients in China: Analysis of social media posts", Journal of Medical Internet Research, May 2020, e19087, https://doi.org/10.2196/19087

[[11] O.D. Apuke, and B. Omar, "Social media affordances and information abundance: Enabling fake news sharing during the COVID-19 health crisis", Health Informatics Journal, July 10, 2021, pp. 1-23, https://doi.org/10.1177/14604582211021470

[[12] S.H. Soroya, A. Farooq, K. Mahmood, J. Isoaho, and S.-e. Zara, "From information seeking to information avoidance: Understanding the health information behavior during a global health crisis", Information Processing \& Management, Elsevier, March 2021, e102440. https://doi.org/10.1016/j.ipm.2020.102440

[13] M. Chong, and H.W. Park, "COVID-19 in the Twitterverse, from Epidemic to Pandemic: Information-sharing behavior and Twitter as an information carrier", Scientometrics, June 23, 2021, pp. 6479-6503 https://doi.org/10.1007/s11192-02104054-2

[[14] A. Abd-Alrazaq, D. Alhuwail, M. Househ, M. Hai, and Z. Shah, "Top concerns of tweeters during the COVID-19 pandemic: A surveillance Study", Journal of Medical Internet Research, April 2020, e19016, https://doi.org/10.2196/19016

[[15] H. Zhao, S. Fu, and X. Chen, "Promoting users' intention to share online health articles on social media: The role of confirmation bias", Information Processing \& Management, November 2020, e102354, https://doi.org/10.1016/j.ipm.2020.102354

[[16] O. Abramova, K. Batzel, and D. Modesti, "Coping and regulatory responses on social media during health crisis: a large-scale analysis", in Proceddings of the 55 th Hawaii International Conference on System Sciences (HICSS-55), ScholarSpace, 2021.

[17] E.A. Skinner, and M. Zimmer-Gembeck, "Coping", Encyclopedia of Mental Health, NeuroScience and Biobehavioral Psychology, https://doi.org/10.1016/B978-012-397045-9.00036-7 
[18] M. Knittel, and R. Wash, "How an Online Health Support Community Responded to the COVID-19 Crisis", in
Proceedings of the $55^{\text {th }}$ Hawaii International Conference on System Sciences (HICSS-55), ScholarSpace, 2021. 